\title{
Hospital Registration Process Reengineering Using Simulation Method
}

\author{
Qiang $\mathrm{Su}^{\dagger^{*}}$, Xiaoyun $\mathrm{Yao}^{\dagger}$, Ping Su${ }^{+}$, Jinghua Shi \\ ${ }^{\dagger}$ School of Economics \& Management, Tongji University, Shanghai 200092, China \\ ${ }^{*}$ NCPC Worker's Hospital, Shijiazhuang, Hebei Province 050011, China \\ ${ }^{+}$Research Center for Healthcare Management, SEM, \\ Tsinghua University, Beijing 100084, China
}

\begin{abstract}
With increasing competition, many healthcare organizations have undergone tremendous reform in the last decade aiming to increase efficiency, decrease waste, and reshape the way that care is delivered. This study focuses on the operational efficiency improvement of hospital's registration process. The operational efficiency related factors including the service process, queue strategy, and queue parameters were explored systematically and illustrated with a case study. Guided by the principle of business process reengineering (BPR), a simulation approach was employed for process redesign and performance optimization. As a result, the queue strategy is changed from multiple queues and multiple servers to single queue and multiple servers with a prepare queue. Furthermore, through a series of simulation experiments, the length of the prepare queue and the corresponding registration process efficiency was quantitatively evaluated and optimized.
\end{abstract}

Key words: Healthcare service; Queuing strategy; Waiting time; Medmodel

\section{INTRODUCTION}

Currently, one of the most concerned issues in Chinese health service is the so called "three longs and one short" problem [1]. The "three longs" represents the long waiting time for registration, the long waiting time before seeing a doctor, and the long waiting time for buying drugs. Contrary to the "three longs" that may add up to two hours, "one short" indicates that the consult time with physicians is very short, usually around 3 to 5 minutes.

This problem mainly results from the large population and scarce healthcare resources. According to statistics, there are about 17,000 hospitals in mainland China. Among them, 977 are the third-class (the highest level hospital with more than 500 beds) hospitals, 5198 are the second-class (the mid level hospital with 100-500 beds), 2574 are the first-class (the lowest level hospital with less than 100 beds), and the rest are small clinics located in township or rural areas [2]. These resources are too limited to take care of such a huge population of more than 1.3 billion. Moreover, most Chinese patients do not trust the lower level hospitals and clinics. They prefer to go to the third-

*Corresponding author: Qiang Su, PhD, Professor, School of Economics \& Management, Tongji University, 1239 Siping Rd., Shanghai 200092, China

Email: servicequality@163.com 
or second-class hospitals even with a minute health issue. As a consequence, on average, each second- or third-class hospital serves more than 2,000 outpatients per day, and this figure can reach 10,000 for some big hospitals located in large cities such as Beijing, Shanghai, and Guangzhou. In these big hospitals, the registration lobby is often crowded with a swarm of outpatients who have to line up in long queues and wait a long time for registration. In some extreme cases, registration may take several hours or even several days. Such scenario and experience seriously jeopardize hospital service quality and patients' satisfaction.

In addressing the long waiting time for registration, this paper is focused on the efficiency improvement of the hospital registration process. The registration related factors of a real hospital, including the service process, queue strategy, and queue parameters were explored systematically in this study. Business process reengineering (BPR) and simulation analysis are employed in the process redesign and performance optimization.

In this paper, literature on BPR and its applications in healthcare are reviewed in section 2. In section 3, the current registration process of the hospital is analyzed and the corresponding operational data are studied. In section 4, the simulation model of the current registration process is established and the simulation analysis is conducted. Thereafter, in section 5, a reengineered process is developed and optimized through simulation experiments. Finally, discussion is given in section 6 , followed by conclusions.

\section{LITERATURE REVIEW ON HEALTHCARE REENGINEERING}

Hammer and Champy defined "business process reengineering" as "the fundamental rethinking and radical redesign of business processes to achieve dramatic improvements in critical, contemporary measures of performance, such as cost, quality, service, and speed" [3]. Chan and Choi proposed a standard BPR analysis procedure based on the existing literature [4]. In this procedure, four steps are included: (1) analyzing the current business process, (2) identifying deficiencies, (3) designing new processes, and (4) forecasting the improvements.

Facing extreme pressures of rapidly changing expectations and constantly increasing financial demands, healthcare organizations have undergone tremendous changes in the last decade to increase efficiency, decrease waste, and reshape the way that care is delivered. BPR has become an increasingly significant and integral part of healthcare systems [5,6]. An investigation of 215 U.S. and Canadian hospital executives indicated that BPR can improve service quality and financial performance, and most of the hospital executives expected the amount of BPR activities in their organizations to increase somewhat/significantly more over the next five years [7].

So far, many healthcare reengineering efforts were focused on the emergency department (ED) and pediatric programs. For instance, Capitulo and Silverberg described how a hospital redesigned its traditional provider-focused obstetric and pediatric program into a patient-focused, family-centered, maternal-child healthcare center [8]. The process, opportunities, challenges, and outcomes of this work demonstrated that a scholarly, data-driven, patient-focused process could result in improved quality and increased patient and staff satisfaction [8]. In reengineering effort of the order-delivery process at the pediatric clinic of a university hospital, Lundgren 
and Heldrup found that the development of modern information technology, along with the developing of standards for communication protocols and the message structure for the electronic interchange of information (EDI) could provide the tools to change the workflow of the traditional order and delivery activities [9].

In terms of the hospital registration process reengineering, Tsai et al. investigated the effect of an electronic hospital information system on nursing quick registration and triage times of an ED in a public teaching hospital [10]. Kesler elaborated a redesign of a pediatric hospital's triage and registration area of the ED of Primary Children's Medical Center in Salt Lake City, Utah [11]. In this case, with the enactment of HIPAA (Insurance Portability and Accountability Act) and the increase in volume of patients, the expansion of the triage area was required. To fulfill this requirement, a multifunctional team was assembled and the reengineering solutions were derived by brainstorming the potential problems that could cause delays in the triage and registration process. Based on existing knowledge in the literature and other hospitals' experience, improvement measures focusing on the layout and facilities redesign were proposed and implemented [11].

Currently, besides the information technologies, the most widely used analysis methods in BPR are subjective approaches such as benchmark, brainstorm, and experience based judgment. More scientific approaches or tools that can be utilized to provide specific and quantitative solutions are highly desirable. To this end, Kwak and Lee explored the multi-criteria mathematical programming (MCMP) method and suggested to utilize this algorithm on effective resources allocation in a BPR planning of a healthcare system [12]. Zakarian and Kusiak emphasized that a structural modeling method and a dynamic simulation tool were very important in the evaluation of the impact of changes and in modeling performance of the proposed process [13]. Vergidis et al. stated that simulation could provide the necessary means to analyze a business process and to identify its bottlenecks so as to provide a solid basis for improving the process [14]. According to [15], many problems of business processes have similarities in terms of project management or production process planning. Therefore, the simulation analysis that has been successfully applied in industry may be applied to healthcare organizations to facilitate their BPR attempts.

The case study in [16] demonstrated how discrete event simulation technique can be used to optimize the use of catheterization capacity. In that work, three experiments were formulated, modeled and simulated to investigate alternative ways to optimize the use of the catheterization room. Wang et al. resolved the overlong waiting time in emergency services using modeling and discrete event simulation with software systems of ARIS and Arena [17]. Raikundalia et al. described a framework and mechanism where simulation models can be developed and applied to improve the access, demand and resource management in hospitals [18]. Khadem et al. studied on the facilities layout optimization [19]. Using the simulation modeling, the layout of the ED of a public hospital was revised and the average waiting time reduced by $75 \%$ and the capacity of the department increased by $10 \%$ [19]. Similarly, the process of the emergency center (EC) of Carondelet St. Mary's Hospital was improved based on simulation. As a result, the EC length of stay was reduced by $7 \%$, the EC monthly volume increased by $5 \%[20]$. 
The above works suggested that BPR is an important approach for a hospital to make significant improvement and to maintain competitive. Meanwhile, simulation analysis can provide a scientific and quantitative methodology for performance evaluation and optimization. However, few simulation-based BPR studies have been conducted focusing on the hospital registration process which is critical for solving the "three longs and one short" problem in China. Under this situation, in this paper, the registration process of a Chinese public hospital is analyzed and reengineered based on a series of simulation experiments.

\section{THE CURRENT REGISTRATION PROCESS}

The study hospital is a second-class public hospital located in Shanghai, China. There are around 2,000 outpatient visits each day to this hospital. Based on field investigations, the flowchart and performance data of the registration system were obtained.

\subsection{The flowchart of the current registration process}

Figure 1 illustrates the flowchart of the current registration process in the study hospital. This registration system consists of three registration counters and three corresponding queues. According to the hospital's regulation, patients must be pre-checked before registering. After the pre-check, the patient gets a ticket on which the suggested consult department name is printed. With the ticket, patient can line up for registration. Although this process is simple, many patients still forget to do pre-check and directly go to the registration counter without the pre-check ticket. In this situation, patients will not be able to register and have to turn back to redo pre-check.

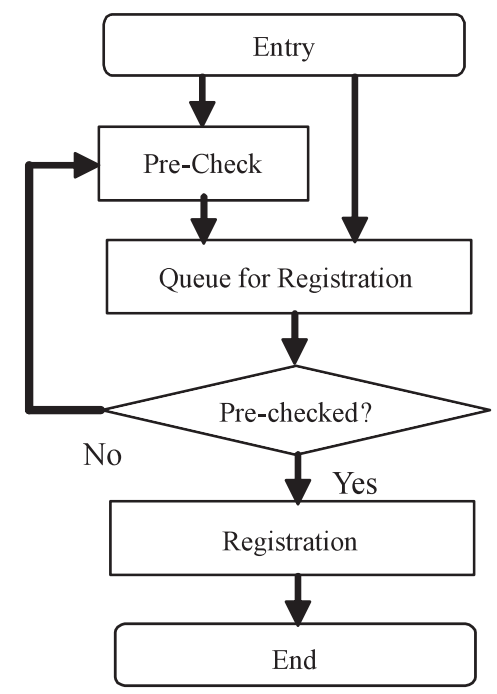

Figure 1. Flowchart of the current registration process 
In Chinese hospitals, especially in big hospitals, in order to ensure the health service quality, the patients are required to do the pre-check before registration. If the pre-check is omitted, the patients might be assigned to an unsuitable doctor or even wrong department since the registration staffs are not healthcare professionals. However, many patients are not aware of the importance of the pre-check. They usually directly go to the registration counter after entering the hospital.

\subsection{Performance data of the current process}

Patient perception of service quality is a function of waiting time, among other factors $[21,22,23]$. It is almost a universal rule that patients do not like to wait. Hence, the total time from entering the hospital to finishing the registration can be employed to measure the efficiency of the registration system and evaluate the service quality. Therefore, the average and maximum total time for registration was measured and statistically analyzed based on field investigations.

The field collected data illustrate that the total time for registration will increase rapidly from several minutes in early morning to more than three quarters at noon. To find out the root causes for the long waiting time, some detailed parameters such as precheck time, registration time, and percentage of patients who forget to go through precheck before registration were analyzed based on extensive investigations in the study hospital. To facilitate the data collection, a computer program was developed using Visual Basic 6.0 incorporated with Access 2003 database system. With this program, the time of events can be automatically recorded by the computer and stored in the database simply by clicking the Enter key.

Five investigators were trained for the data collection. One was responsible for recording patients who came to hospital for registration. The number of patients and their arrival times were recorded. Two investigators were assigned to record the precheck time and registration time respectively. Meanwhile, another investigator was assigned to follow the patients from their entry until departure, so that the total time for registration could be obtained. The fifth investigator was in charge of counting how many patients forgot to do pre-check before registration.

Based on the collected data, we found that the pre-check time ranges from 3 seconds to 48 seconds with an average of 10 seconds, and the registration time was spread out quite widely. Most patients could finish registration in less than 30 seconds, but for some elder patients, the registration time lasted for 1 and a half minutes. On the contrary, for young patients with Medicare card, the registration time was less than 10 seconds since they did not need to pay cash at the registration counter. Meanwhile, we found that approximately $60 \%$ of patients forgot to do pre-check.

\section{SIMULATION OF THE CURRENT PROCESS}

MedModel was utilized in the simulation analysis of this study. MedModel is a Windows-based simulation program dedicated to the special needs of the healthcare industry [12,21]. According to the classification in [24], our simulation model can be characterized as a queue model. 


\subsection{Model construction}

The simulation model was established as follows.

\section{1) Analysis of time distribution}

To simulate the dynamic and stochastic process, the distribution of patients' inter-arrival time, the distribution of pre-check time, and the distribution of registration time should be evaluated first.

Based on the field-collected data, these distributions were derived using the function Stat::Fit in MedModel. The inter-arrival time data in Figure 2 demonstrates an exponential distribution. However, the average inter-arrival time $\lambda$ is not a stationary constant. It varies from 6.5 seconds to 12.5 seconds (see Figure 3 ). The exponential distributions of the pre-check time and the registration time are shown in Figure 4 and Figure 5 respectively.

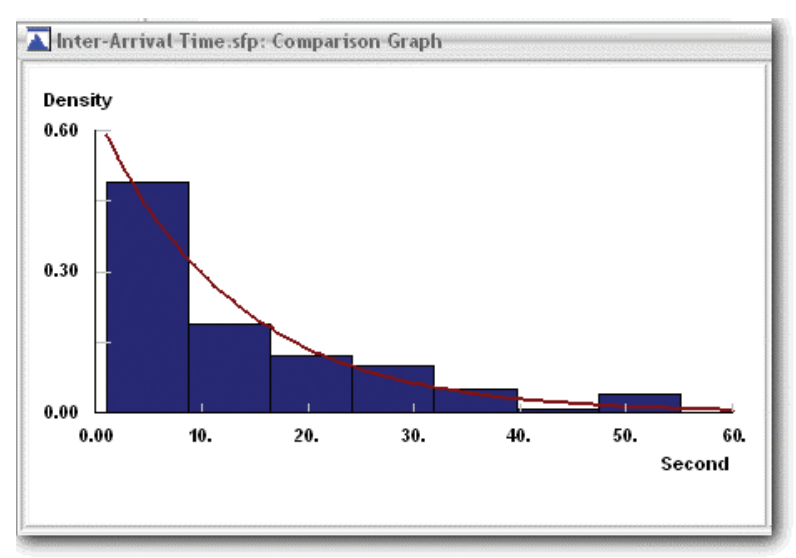

Figure 2. The distribution of the inter-arrival time

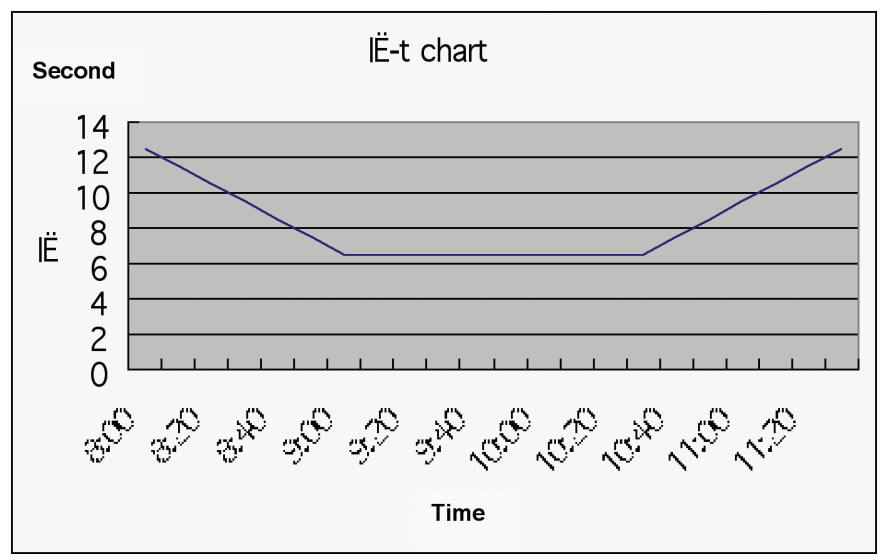

Figure 3. The change of average inter-arrival time $\lambda$ 


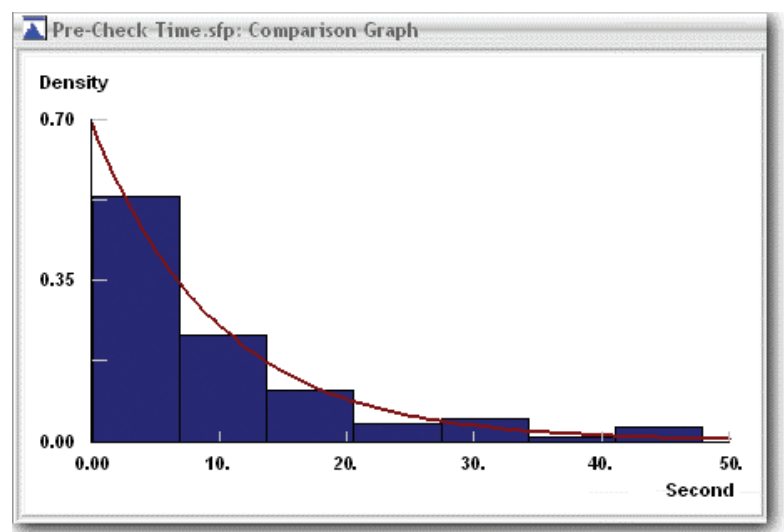

Figure 4. The distribution of pre-check time

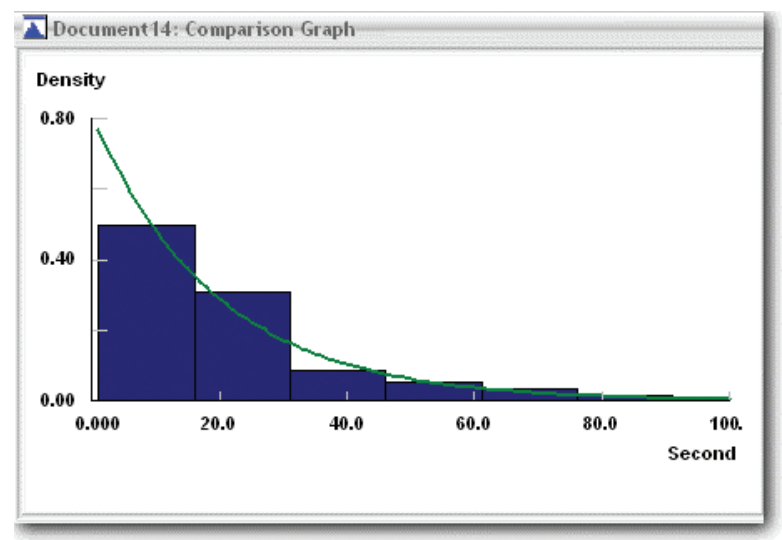

Figure 5. The distribution of the registration time

\section{2) Definition of locations}

In this model, the locations of entry, pre-check queue, pre-check, queues for registration, registration, and departure, were defined. The distances between different locations were also defined so that the walking time between locations could be taken into consideration in the simulation analysis.

\section{3) Definition of entities}

Three kinds of entities namely patient, time_adjuster, and time_counter were defined in this work. The patient entity represents a patient who enters the registration system. The entity time_adjuster was defined and used to adjust the average inter-arrival time $\lambda$ in every 10 minutes according to the curve in Figure 3. The entity time_counter was defined and utilized to record the duration (in second) of a process. 
4) Construction of the simulation model

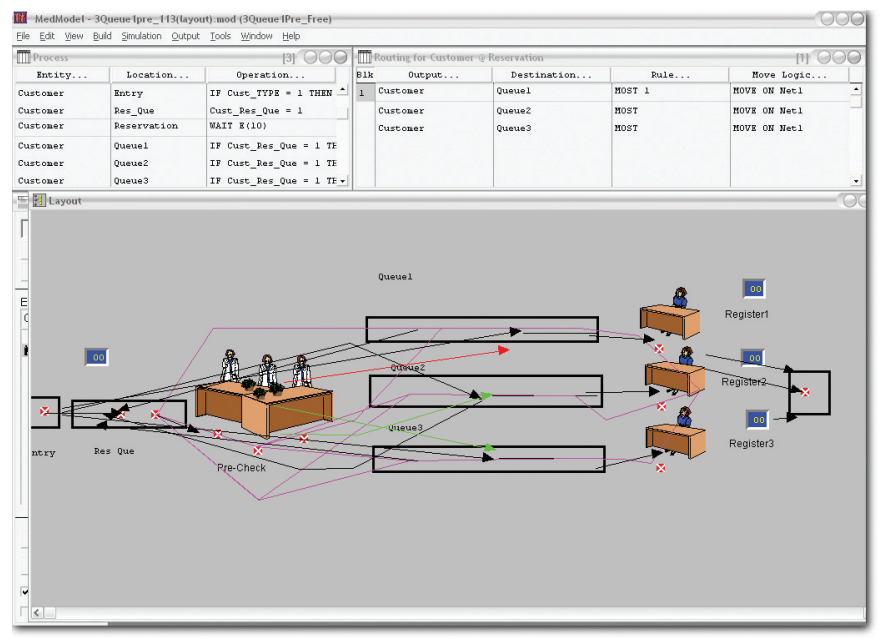

Figure 6. Simulation model for the current registration process

As shown in Figure 6, the locations are connected by arrowed lines according to the logic and constraint of the process. The directed network denotes the pathway of the patient flow in the study hospital with the current process. In simulation, patient entities can flow from one location to the subsequent locations along with the arrowed lines. In the same time, the time needed for each location can be obtained and the total registration time can be derived accordingly.

\subsection{Simulation analysis}

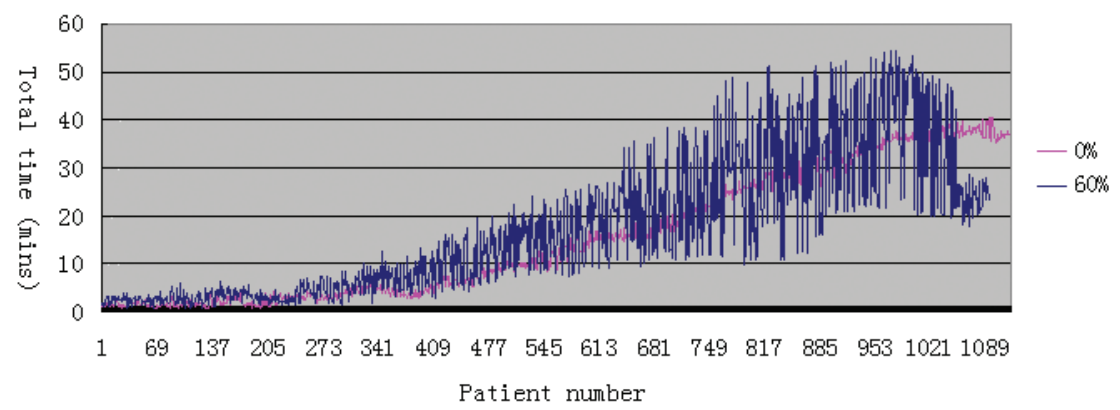

Figure 7. Total time for registration

To validate the correctness of the simulation model, a three-hour period (from 8:00 a.m. to 11:00 a.m.) of the current process was simulated. As shown in Figure 7, a total of 1089 patient entities were generated in this period of time. Among these patients, $60 \%$ were randomly selected to directly go to the registration queue without pre- 
checking. The blue curve illustrates the change of the total registration time during this period of time. As more and more patients entering the registration system, the total registration time for a patient increases rapidly from several minutes to more than 50 minutes. These findings fairly agree with the observed times in field study.

With this model, an improved situation was simulated in which no patient forgot precheck before registration. The simulation result is shown as the pink curve in Figure 7. The average and maximum total times of these two simulations $(60 \% \mathrm{vs} .0 \%)$ are listed in Table 1.

Table 1 The average and maximum total times ( $0 \%$ vs. $60 \%$ of patients who forget pre-check)

\begin{tabular}{lcccc}
\hline Time period (a.m.) & \multicolumn{2}{c}{ If 60\% forget pre-check } & \multicolumn{2}{c}{ If 0\% forget pre-check } \\
\hline & $\begin{array}{c}\text { Average total } \\
\text { time (min) }\end{array}$ & $\begin{array}{c}\text { Maximum total } \\
\text { time (min) }\end{array}$ & $\begin{array}{c}\text { Average total } \\
\text { time (min) }\end{array}$ & $\begin{array}{c}\text { Maximum total } \\
\text { time (min) }\end{array}$ \\
\hline 8:00-11:00 & 17.24 & 54.62 & 15.77 & 40.57 \\
8:00-09:00 & 4.14 & 12.70 & 2.68 & 5.95 \\
9:00-10:00 & 22.19 & 52.92 & 17.98 & 36.02 \\
10:00-11:00 & 33.37 & 54.62 & 36.96 & 40.57
\end{tabular}

The data in the first row demonstrate that, when each patient does pre-check before registration, the average and maximum total registration time can be reduced by $8.5 \%$ and $25.7 \%$, respectively. This implies that the registration process should be redesigned to ensure that every patient does pre-check before registration.

\section{REDESIGN OF THE REGISTRATION PROCESS}

The field-collected data demonstrate that the time spent on pre-check and registration only accounts for $3 \%$ of the total time. Most of the time is spent on queuing for precheck or registration.

To solve the long queuing time problem, a Root Cause Analysis was conducted to identify the potential improvement approaches. As shown in Figure 8, five facets, e.g., people, machine, material, method and environment, are evaluated and 3 to 4 factors are identified for each facet. For instance, in terms of people facet, three different kinds of participants, e.g., triage nurse, register and patient, are involved in this process. We may improve the process efficiency by providing better training to these people. However, it is difficult to evaluate quantitatively the effectiveness of training with the simulation calculation.

For the facet of machine, the improvement possibility is very limited since the computers and printers are already networked and the workflow is already streamlined in the case hospital. In terms of material facet, the related materials are trivialities and completely determined by the method of registration which is a factor in the method facet. In some private hospitals, each patient is issued a membership card and the registration fee can be automatically deducted from the deposit account by the Hospital Information System (HIS). Thus, patients do not need to go through registration before seeing a doctor. However, this approach is not available in the case hospital since it is a public institute. 
The above analysis suggests that we should focus on the factors of pre-check, queue strategy, buffer queue in the method facet. The factor of pre-check has been evaluated in section 4. In this section, the queue strategy and the buffer queue will be designed and evaluated with simulation tool. The layout design in environment facet will be considered when necessary.

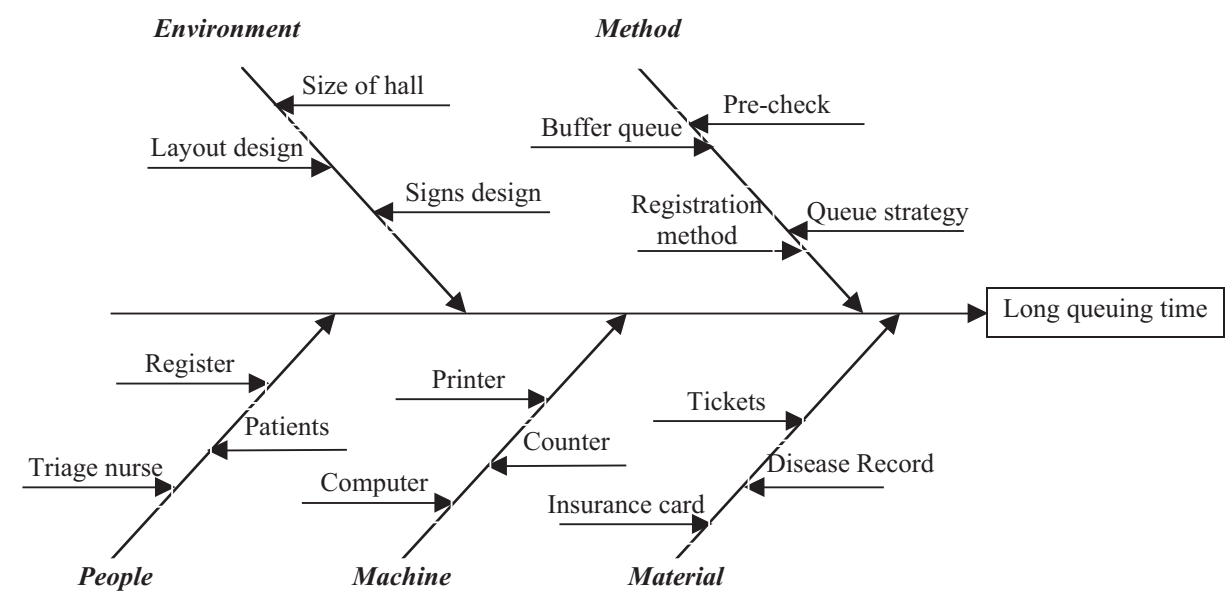

Figure 8. Root cause analysis

\subsection{The new registration process}

According to Kaboudan [25], due to the simplicity and efficiency, the multi-server and single queue system is practically superior to the multi-queue and multi-server system. With this point in mind, the original queue strategy was replaced by a single queue and multi-server strategy and the patients would be served on the first come first serve (FCFS) basis. Meanwhile, to save walking time, a buffer queue (prepare queue) was designed near the registration counters.

The flowchart of the reengineered registration process is exhibited in Figure 9. In the new process, patients must first line up for pre-check. After finishing the pre-check, patients receive tickets for registration. On the ticket, in addition to the suggested consulting department name, a sequence number is printed for registration. With the ticket in hand, most patients can be seated to wait for registration service.

To save walking time from seat to the registration counter, a certain number of patients will be required to line up in the buffer queue (prepare queue) near the registration counters. The first patient in the prepare queue will be served by the next available register. In the meantime, a seated patient will be called to join the prepare queue according to his/her sequence number.

The layout design of the new process is illustrated in Figure 10. Arrays of chairs are set up in the registration hall so that patients can be seated when waiting for registration. Meanwhile, a prepare queue is set up near the registration counters to improve the registration efficiency. 


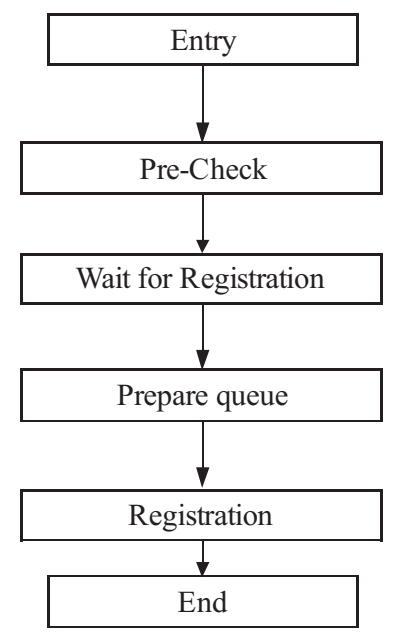

Figure 9. The redesigned registration process

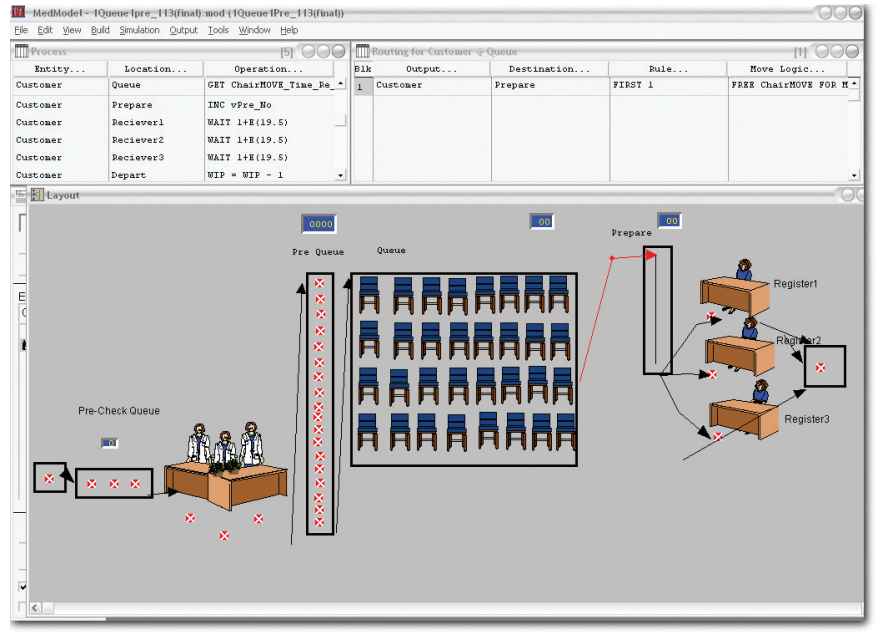

Figure 10. The simulation model of the new registration process

\subsection{Simulation experiments}

The size of the prepare queue was determined through a series of simulation experiments. Six experiments with different prepare queue lengths of $0,5,10,15,20$, and 25 persons were conducted. 
Table 2. Simulation experiments of new process

\begin{tabular}{cccc}
\hline $\begin{array}{c}\text { Capacity of the prepare } \\
\text { queue (Persons) }\end{array}$ & $\begin{array}{c}\text { Avg. total } \\
\text { time (min) }\end{array}$ & $\begin{array}{c}\text { Avg. time on } \\
\text { seat (min) }\end{array}$ & $\begin{array}{c}\text { Avg. number of } \\
\text { patients in system }\end{array}$ \\
\hline 0 & 39.08 & 6.96 & 321.54 \\
5 & 24.72 & 5.06 & 194.21 \\
10 & 3.96 & 1.97 & 29.45 \\
15 & 3.18 & 1.18 & 24.15 \\
20 & 3.15 & 0.92 & 23.68 \\
25 & 3.15 & 0.67 & 23.68 \\
\hline
\end{tabular}

As shown in table 2, the average total time is 39.08 minutes when the length of the prepare queue equals 0 (no prepare queue). This finding indicates that the total time is the maximum without the prepare queue. In other words, the walking time significantly affects the efficiency of the registration system. The total time decreases drastically with the increase of the prepare queue size. When the prepare queue length is more than or equal to 20, the total time becomes a constant of 3.15 minutes. Meanwhile, the average time on seat will decrease with the increase of the prepare queue length.

To achieve the highest service quality, the total time should be minimized whereas the time on seat should be maximized. Under this consideration, referring to data in Table 2, the optimal prepare queue length should be between 15 and 20 persons. Thereafter, a series of fine-tuning simulation experiments were carried out to find out the optimal prepare queue length.

Table 3. Fine-tuning simulation experiments

\begin{tabular}{cccc}
\hline $\begin{array}{c}\text { Length of the prepare } \\
\text { queue (Persons) }\end{array}$ & $\begin{array}{c}\text { Avg. total } \\
\text { time (min) }\end{array}$ & $\begin{array}{c}\text { Avg. time on } \\
\text { seat (min) }\end{array}$ & $\begin{array}{c}\text { Avg. number of } \\
\text { patients in system }\end{array}$ \\
\hline 15 & 3.18 & 1.18 & 24.15 \\
16 & 3.15 & 1.13 & 23.68 \\
17 & 3.15 & 1.08 & 23.68 \\
18 & 3.15 & 1.02 & 23.68 \\
19 & 3.15 & 0.97 & 23.68 \\
20 & 3.15 & 0.92 & 23.68 \\
\hline
\end{tabular}

Table 3 shows that the best performance can be achieved when the prepare queue length is 16 persons. In this circumstance, patients just need to wait on average 3.15 minutes for the whole process, and during this period of time, they can be seated for 1.13 minutes on average.

Figure 11 depicts the total time of three simulation results with different prepare queue sizes (PQS) of 10, 15 and 20 persons. Compared with the original process, the redesigned process can reduce the maximum total time for registration from more than 50 minutes to 8 minutes. 


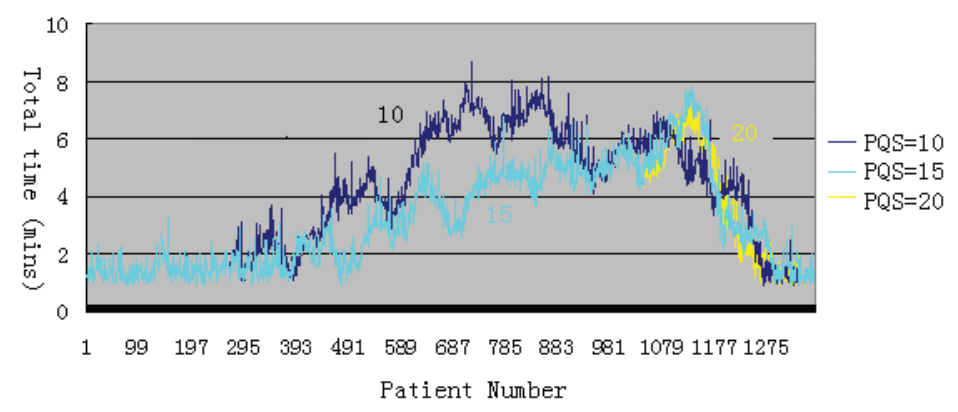

Figure 11. The total time for different prepare queue sizes (PQS)

\section{DISCUSSION}

BPR has been extensively applied in healthcare industry for more than ten years [7]. However, most of the related practices heavily depend on subjective approaches such as brainstorm, benchmark, and experience-based judgment [11]. To facilitate BPR analysis and to make the reengineering more scientific, utilization of simulation analysis should be encouraged.

The above analysis results demonstrate that the simulation modeling can provide essential assistance in the healthcare service process evaluation and reengineering. With the simulation analysis, the critical factors that determine the healthcare service efficiency can be readily identified. For the case hospital, the calculation results illustrate that the rate of patients who forget to pre-check, the queue strategy for registration, and the length of the prepare queue are the most important factors for shortening the overlong waiting time. Moreover, with the simulation experiments, the different feasible improvement approaches or strategies can be appraised and compared quantitatively. For the case hospital, the simulation results verify that the most critical factor for the service efficiency is the length of the prepare queue. The second one is the rate of patients who forget to pre-check. With these conclusions, a more scientific and optimal process reengineering resolution can be achieved for the healthcare service improvement. The layout of the hospital's outpatient registration hall should be redesigned (with assistance of some instruction boards and signs) so as to ensure that patients pre-check before registration. Arrays of chairs should be set up in the registration hall for patients to sit while waiting for registration. More importantly, a prepare queue with a capacity of 16 persons should be set up near the registration counters.

Although simulation analysis is a useful tool for BPR, we should be aware of its shortcomings. Generally speaking, a commercial simulation system is too complex to be mastered easily and applied properly. To construct a simulation model, the event generator, the definitions of entities and locations, the logic and constraint design should be considered very carefully. For simulation of a complicated process, difference between the simulation result and the real operational performance cannot be avoided. Furthermore, if substantial changes have been made in process logic, the validation of the simulation model will become very difficult since fewer elements can be tested by the actual data collected from the existing process. 
Besides the technical issues, some socio-cultural factors should also be taken into consideration in healthcare system's reengineering. Walston, Burns, and Kimberly suggested that steering committees, project teams, codification of the change process, and executive involvement in core changes could modify the results of reengineering to improve an organization's competitive position [26]. Kroposki and Murdaugh, et al., noted that the hospital reengineering projects could be significantly affected by the role conflict, role ambiguity, organizational commitment, and job satisfaction experienced by clinical team members [27]. On the other hand, reengineering of a healthcare organization would generate impacts on job satisfaction, professional identity and other work related attitudes [28].

\section{CONCLUSION}

In this paper, a public hospital's registration process is reengineered based on the BPR principle and simulation analysis. The case study demonstrates that simulationsupported BPR can provide effective help to health care process reengineering. This method can also provide guidance in the design and optimization of new healthcare systems. Many issues are still open for further investigation. For instance, the number of registers, the number of chairs, the layout of the facilities, and the patient walking speed should be studied in more details.

\section{ACKNOWLEDGMENT}

This research was sponsored by National Nature Science Foundation of China (70672077, 70832005), Medical \& Engineering Interdisciplinary Research Foundation of Shanghai Jiao Tong University (YG2007ZD10), and sponsored by Research Center for Healthcare Management, SEM, Tsinghua University.

\section{REFERENCES}

[1] http://web.peopledaily.com.cn/shidai/10/newfiles/a1030.html

[2] X. Zhang. Application of the resources of the retailers, Newspaper of Chinese Medicine, Apr. 7, 2005.

[3] M. Hammer, J. Champy. Reengineering the corporation - A manifesto for business revolution. Nicholas Brealey. (1993) P32-36.

[4] Stephen L. Chan and Chung For Choi. A conceptual and analytical framework for business process reengineering. International Journal of Production Economics, 1997, 50, 211-223.

[5] L.D. Urden, S.L. Walston, Outcomes of hospital restructuring and reengineering - How is success or failure being measured? Journal of Nursing Administration, (2001) 31(4): 203-209.

[6] J.R. Caron, S.L. Jarvenpaa, D.B. Stoddard, Business reengineering at CIGNA Corporation: Experiences and lessons from the five years. MIS Quarterly 18(3), (1994) 233-250.

[7] Shih-Jen Kathy Ho, Lilian Chan, and Roland E. Kidwell Jr. The implementation of business process reengineering in American and Canadian hospitals. Health Care Management Review, 1999, 24(2): 19-31.

[8] Capitulo, K L; Silverberg, M C. Creating patient-focused, family-centered, maternal-child and pediatric healthcare. MCN, the American Journal of Maternal Child Nursing, 2001, 26(6):298-3055

[9] Lundgren, P A; Heldrup, J. A case study: business process re-engineering (BPR) of the order-delivery process at the pediatric clinic at the University Hospital of Lund. Medinfo, 1995, 8(1), 543-554.

[10] J. Tsai, A.T. Limkakeng, R.R. Roberts, et al., Impact of institution of an electronic hospital information system on nursing quick registration and triage times, Annals of Emergency Medicine 2004, 44 (4): 122. 
[11] Kesler, CJ. Redesign of a pediatric hospital's triage and registration area. Journal of Emergency Nursing, 2007, 33(3): 223-227.

[12] N.K. Kwak, C.W. Lee, Business process reengineering for health-care system using multicriteria mathematical programming, European Journal of Operational Research, 2002, 140 (2): 447-458.

[13] A. Zakarian and A. Kusiak, Process analysis and reengineering, Computers \& Industrial Engineering 2001, 41: 135-150.

[14] Kostas Vergidis, Ashutosh Tiwari, and Basim Majeed. Business Process Analysis and Optimization: Beyond Reengineering. IEEE Transactions on System, Man, and Cybernetics - Part C: Applications and Reviews, 2008, 38(1): 69 -82.

[15] P. Volkner and B. Werners. A decision support system for business process planning. European Journal of Operations Research, 2000, 125(3): 633-647.

[16] S. Groothuis, G.G. van Merode, and A. Hasman, Simulation as decision tool for capacity planning, Computer Methods and Programs in Biomedicine, 2001, 66(2-3):139-151.

[17] Wang, Tao; Guinet, Alain; Belaidi, Aissam; Besombes, Beatrix. Modelling and simulation of emergency services with ARIS and Arena. case study: The emergency department of Saint Joseph and Saint Luc hospital. Production Planning and Control, 2009, 20(6): 484-495.

[18] Raikundalia, Gitesh K.1; Bain, Christopher A.2; Mehta, Snehal3. Towards an advanced computing solution for hospital management using discrete event simulation. International Review on Computers and Software, 2009, 4(1):15-25.

[19] Khadem, Mohammed; Bashir, Hamdi A.; Al-Lawati, Yasin; Al-Azri, Fatma. Evaluating the layout of the emergency department of a public hospital using computer simulation modeling: A case study. 2008 IEEE International Conference on Industrial Engineering and Engineering Management, IEEM 2008, p 1709-1713

[20] Ferrin, David M.; Miller, Marty J.; McBroom, Diana L. Maximizing hospital financial impact and emergency department throughput with simulation. Proceedings of the 2007 Winter Simulation Conference, WSC, 2007: 1566-1573.

[21] C.R. Harrell and R.N. Price, Healthcare Simulation Modeling and Optimization Using Medmodel, Proceedings of the 2000 Winter Simulation Conference, 2000, 203-207.

[22] W.J. Hopp and M.L. Spearman, Factory Physics: Foundations of Manufacturing Management, (Irwin/ McGraw-Hill, New York, 2000).

[23] Pines JM, Iyer S, Disbot M, Hollander JE, Shofer FS, Datner EM. The effect of emergency department crowding on patient satisfaction for admitted patients. Acad Emerg Med. 2008 Sep;15(9):825-31.

[24] P.A. Fishwick, Simulation Model Design and Execution: Building Digital Worlds, (Prentice Hall, Englewood Cliffs, NJ, 1995).

[25] M.A. Kaboudan, A Dynamic-server Queuing Simulation, Computers \& Operations Research, 1998, 25(6): 431-439.

[26] S.L. Walston, L.R. Burns, J.R. Kimberly, Does reengineering really work? An examination of the context and outcomes of hospital reengineering initiatives, Health Services Research, 2000, 34 (6): 1363-1388.

[27] M. Kroposki, C.L. Murdaugh, A.S. Tavakoli, et al., Role clarity, organizational commitment, and job satisfaction during hospital reengineering, Nursing Connections, 1999, 12 (1): 27-34.

[28] K. Neuman, The effect of organizational reengineering on job satisfaction for staff in hospital social work departments, Social Work in Health Care, 2003, 36 (4): 19-33. 



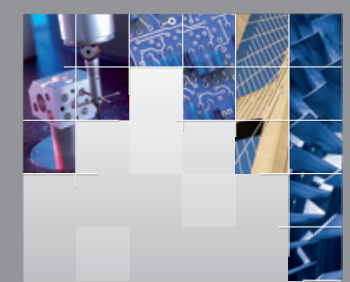

\section{Enfincering}
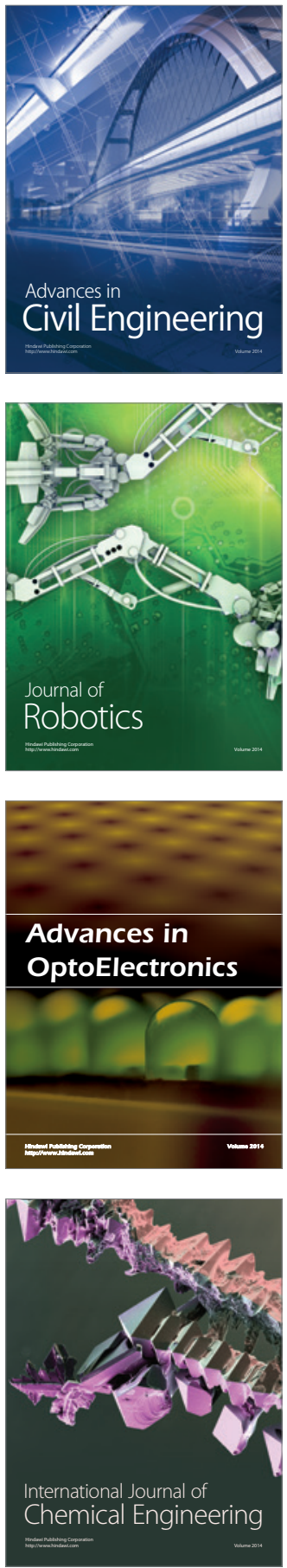

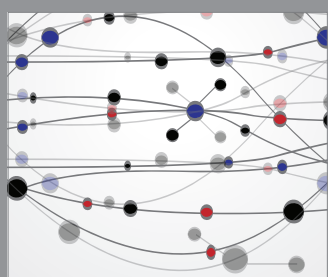

The Scientific World Journal

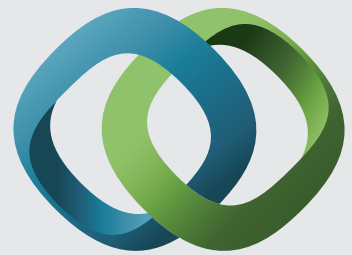

\section{Hindawi}

Submit your manuscripts at

http://www.hindawi.com
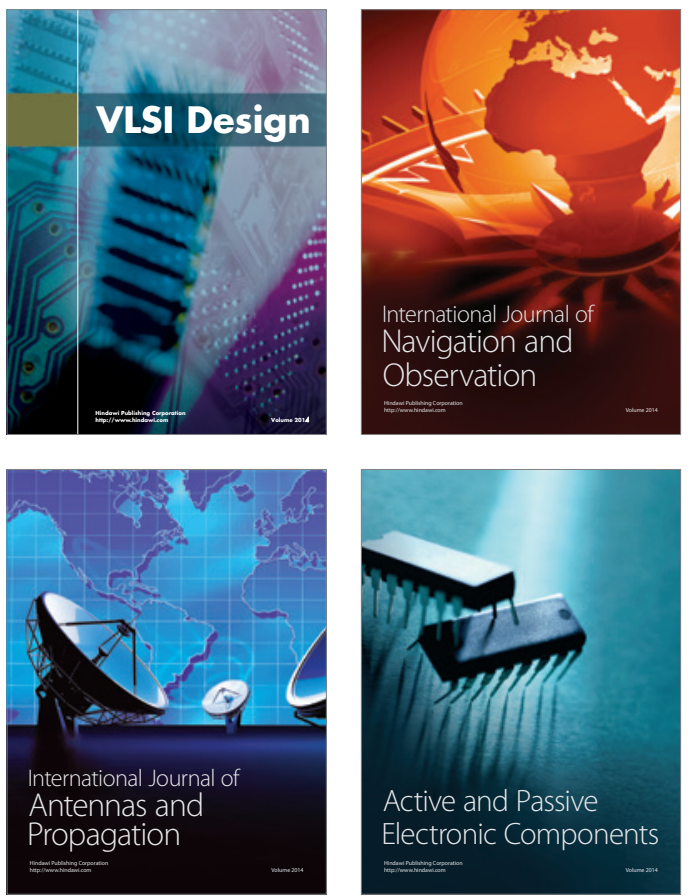
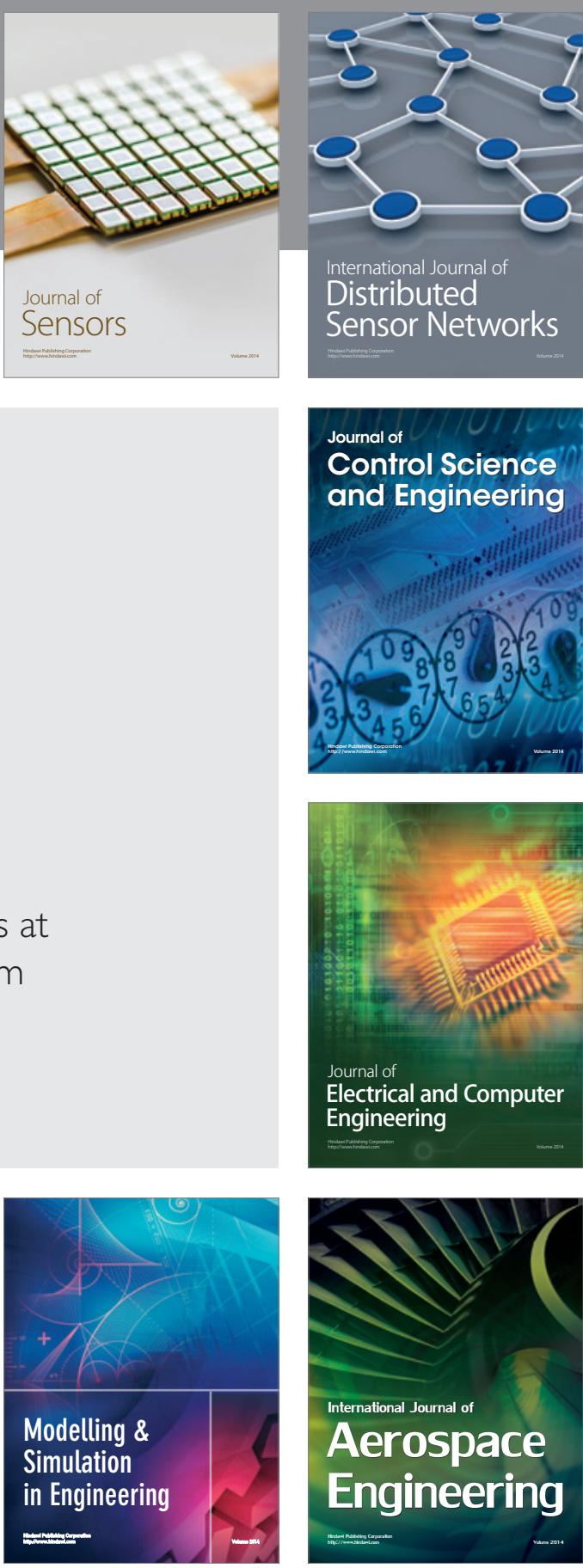

International Journal of

Distributed

Sensor Networks

Journal of

Control Science

and Engineering
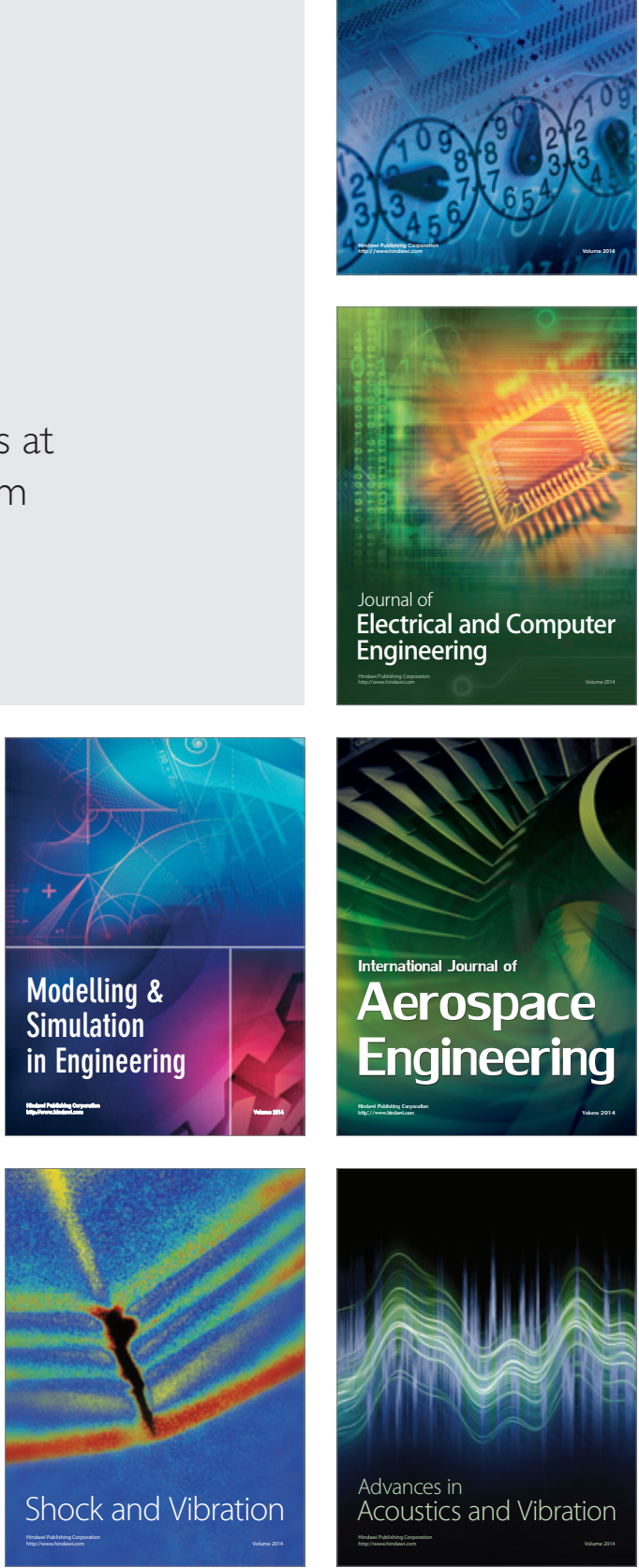